\title{
Estimación del indicador kilómetro vehículo recorrido (KVR) mediante ecuaciones lineales y sus aplicaciones en consumos energéticos de transporte
}

\author{
Juan P. Díaz-Samaniego y Jairo Castillo-Calderón ${ }^{\star}$ \\ Universidad Nacional de Loja, Facultad de la Energía, las Industrias y los Recursos Naturales no Renovables, Loja- \\ Ecuador (correo-e: juan_pablo_diaz2001@yahoo.es; jdcastilloc@unl.edu.ec)
}

* Autor a quien debe ser dirigida la correspondencia.

Recibido Mar. 2, 2021; Aceptado Abr. 29, 2021; Versión final Jul. 7, 2021, Publicado Dic. 2021

\begin{abstract}
Resumen
La presente investigación tiene como objetivo desarrollar una nueva metodología para estimar el indicador KVR (Kilómetro Vehículo Recorrido) y los consumos energéticos por tipo de transporte terrestre. Se realizaron los cálculos para la Provincia de Loja (Ecuador), para el año 2014, mediante un método de balance energético de OLADE (Organización Latinoamericana de Energía). Éste es combinado con un modelo análogo a la solución de sistemas de flujo de Hardy Cross a través de ecuaciones lineales. Se representa al sistema de trasporte como un balance energético, cuyas entradas son: el número y tipo de vehículos, consumo específico promedio de cada grupo vehicular y los despachos globales de combustible. Los resultados muestran una variación del $2 \%$ entre la energía consumida calculada y la energía total despachada. Del total de 1'123.198 BEP, $24,65 \%$ fue destinado para transporte de pasajeros y $66,18 \%$ para transporte de carga. Se concluye que la metodología es apropiada, aplicable a la realidad nacional, y replicable a otras provincias.
\end{abstract}

Palabras clave: combustible; consumo de energía; indicador KVR; método Hardy Cross; parque automotor

\section{Estimation of the indicator vehicle kilometers traveled (VKT) by using linear equations and their applications in transport energy consumption}

\begin{abstract}
The present research study aims to develop a novel methodology for estimating the VKT (Vehicle Kilometers Traveled) indicator and the energy consumption according to road transport type. Calculations are made for the Province of Loja (Ecuador) for the year 2014 by using an OLADE (Latin American Energy Organization) energy balance method. This is combined with a model similar to the Hardy Cross hydraulic flow systems solution with linear equations. The transportation system is represented as an energy balance and the inputs are: total fleet and vehicle type, specific average consumption for each vehicle type, and global fuel sales. The results show that there is a $2 \%$ variation between calculated consumed energy and total dispatched energy. The total energy used is $1,123,198 \mathrm{BEP}$, of which $24.65 \%$ is for passenger transport and $66.18 \%$ for cargo transport. It is concluded that the proposed methodology is appropriate, applicable nation-wide, and replicable in other provinces.
\end{abstract}

Keywords: fuel; energy consumption; VKT indicator; Hardy Cross method; car fleet 


\section{INTRODUCCIÓN}

A nivel mundial la demanda de energía ha ido aumentando progresivamente, de tal forma que a partir de 1970 hasta la actualidad se ha duplicado (Shimoda et al., 2020). Esta tendencia seguirá a menos que se tomen las medidas pertinentes para atenuar este crecimiento. El suministro principal de energía primaria a nivel mundial se da a través del petróleo y gran parte de esta energía está relacionada directamente con la explotación de los sistemas de transporte (Engo, 2019). Los datos disponibles referentes al 2015, indican que el sector del transporte representa el $28.8 \%$ del consumo mundial de energía y el $65.2 \%$ del consumo final total de petróleo (IEA, 2018). Siguiendo esta tendencia, en América Latina y el Caribe, el sector del transporte representó el $36,8 \%$ del consumo total de energía, basándose principalmente en petróleo con $88.4 \%$ del total energía consumida, seguida de biocombustibles, gas natural y electricidad, con $7.8 \%$, 3.5\% y $0.3 \%$, respectivamente. Este incremento continuo del consumo de energía en el sector del transporte está asociado al crecimiento económico de estos países (Rehermann y Romero, 2018), donde el transporte de mercancías en la región, se realiza principalmente por carretera. En Ecuador, el transporte es el sector de mayor consumo de energía (Sierra, 2016; Rocha et al., 2018) mostrando un crecimiento constante a lo largo del tiempo. Para el 2019, el Ecuador tuvo un consumo de energía de 94,4 miles de kBEP, un incremento de 1,36 veces respecto al 2009, donde históricamente el sector transporte es el de mayor consumo y ha incrementado su participación anual, pasando del $36 \%$ en el año 2009 al 49,2\% en el año 2019, esto es 24.947 kBEP y 46.423 kBEP, respectivamente, tal como se muestra en la Fig. 1. Por otro lado, el transporte genera costos elevados en importaciones de combustible, los mismos que están subsidiados para la comercialización interna, y esto induce al consumo de transporte particular por no contar con una adecuada focalización en los sectores económicos y sociales que lo necesita. Lo indicado repercute en ineficiencias del sector transporte, puesto que no se externalizan los verdaderos costos de uso automotriz como: subsidios, salud (enfermedades causadas por gases contaminantes), congestión urbana, tiempo perdido en el tráfico, accidentes, altos costes en infraestructura, etc., todo esto generado por los vehículos en las ciudades.

Una herramienta utilizada en gran parte de los países para cuantificar el uso de los vehículos y sus consecuencias energéticas y ambientales es el indicador KVR (Song et al., 2014). Bajo este contexto, existen algunas investigaciones que tienen como factor común el uso de este indicador. Seethaler y Rose (2009), evaluaron los resultados derivados del programa TravelSmart en el área del gobierno local de Darebin, Australia, que consistía en una iniciativa comunitaria, cuyo objetivo era lograr una reducción del $10 \%$ en los viajes en automóvil y los KVR en la población objetivo. Para ello se aplicó encuestas a los habitantes, antes y después del viaje, que incluían lecturas del odómetro durante una semana. Llegaron a la conclusión que el programa TravelSmart no pudo inducir una reducción estadísticamente significativa en el cambio del comportamiento de viaje y el KVR diario promedio. McIntosh et al. (2014) analizaron el rol de la forma urbana y el tránsito en la dependencia del automóvil, el estudio abarcó a 26 ciudades globales desde 1960 hasta el 2000. El modelo de ecuaciones estructurales realizado indicó que la densidad urbana y la provisión de servicios de tránsito tienen una relación causal con los viajes en vehículos privados y son adicionales a las influencias de la región y la tipología de ciudades. También demostraron que los servicios de transporte ferroviario son los más vinculados a la reducción de KVR per cápita. Por lo tanto, sugieren que las implicaciones políticas para las ciudades que buscan limitar la dependencia del automóvil deberían apoyar la inversión en sistemas de tránsito ferroviario de calidad y construir densidades urbanas a su alrededor, en lugar de aumentar la capacidad vial.

Figliozzi (2007), a través de su investigación, estudia los viajes de vehículos comerciales urbanos en relación con el KVR generado, además, explora el impacto de ciertas redes y cambios de política por tipos de viajes comerciales. Finalmente, discute la recopilación de datos respecto a los viajes vacíos, distribuciones de longitud de viaje (TLD) e implicaciones de modelado de generación/distribución de viajes. Los resultados demuestran que los recorridos multiparada generan más KVR que las entregas directas, incluso para cargas útiles iguales. A medida que el tamaño promedio de los pedidos disminuye y las ventanas de tiempo se acortan, la eficiencia de los recorridos también disminuye. Asimismo, comprueba que el porcentaje de viajes vacíos no tiene correlación con la eficiencia de los tours en cuanto a generación de VKT o número anual de viajes. Estipula que la duración promedio del viaje y la forma del TLD dependen en gran medida del tipo de recorrido, la distancia desde el centro de distribución al área de servicio, la densidad de paradas y el número de paradas por recorrido. Weerasekera y Amarasingha (2017) estiman el KVR mediante encuestas realizadas a viajeros durante 12 meses en la provincia de Sri Lanka. Para ello, registran el modo de viaje, el número total de kilómetros recorridos y el número de pasajeros. Además, recopilan información sociodemográfica que incluye edad, sexo, situación laboral, ingresos y zona residencial. No obstante, los resultados sugieren inexactitud, puesto que los datos dependen de las respuestas subjetivas de los usuarios de la vía que no fueron validados. Además, el estudio se centra únicamente en la estimación del KVR, y no es vinculante con otros objetivos primordiales que persigue la utilización de este indicador, tales como: la estimación de emisiones contaminantes, consumo de energía, análisis de accidentabilidad, impacto del tráfico. 


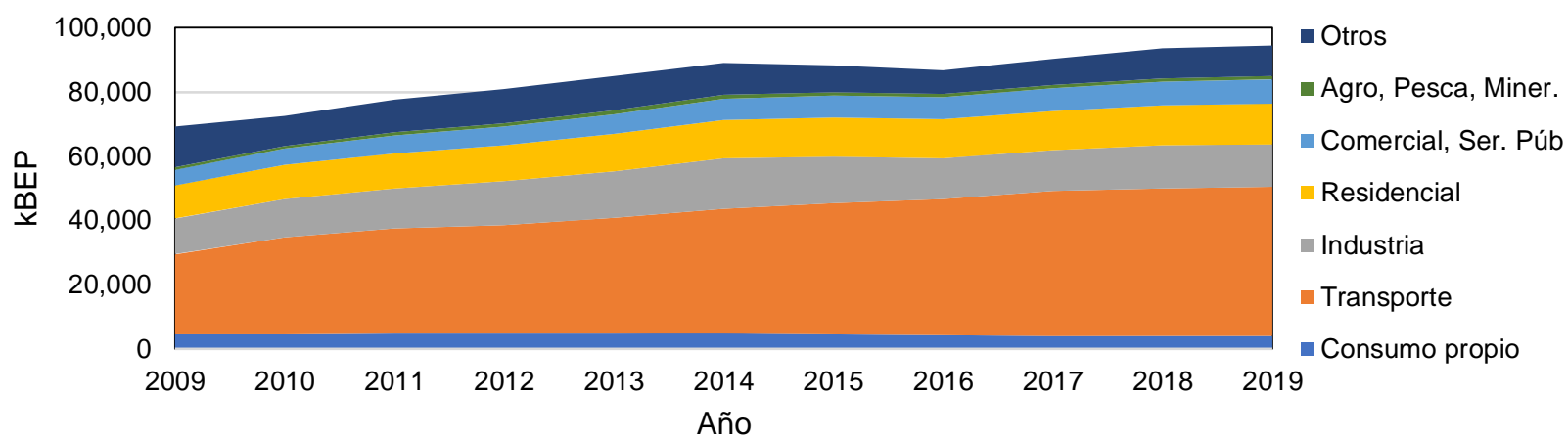

Fig. 1: Desagregación del consumo de energía por sectores (Datos tomados de MERNNR, 2019)

Solís y Sheinbaum (2013) analizan mediante un método ascendente las tendencias de consumo de energía y emisiones de Gases de Efecto Invernadero (GEI) para el transporte terrestre, donde se contaba previamente con la información y estimaciones nacionales KVR y la intensidad de uso de la flota. Es conocido que el consumo de energía para el transporte está directamente relacionado con la emisión de GEI. En la misma dirección, Pérez y Monzón de Cáceres (2008) analizan las tendencias de consumo de energía en España para transporte terrestre con sus respectivas emisiones de GEI, donde se contaba con recorridos promedio. Uno de los análisis más importantes que relaciona los despachos de combustible con los recorridos KVR, es el realizado por el Departamento de Infraestructura y Transporte del Gobierno Australiano, en el estudio de "Vehículo-Kilómetro recorrido por carretera: estimación de las ventas de combustible estatales territoriales", el cual analiza mediante un método simple de comparación la eficiencia de cada grupo vehicular y ventas trimestrales de combustible con las ventas anuales para calcular los recorridos de los diferentes grupos vehiculares de la región y del país (Gargett et al., 2011). Por otro lado, el estudio sobre consumos del sector transporte realizado por Instituto Nacional de Eficiencia Energética y energías Renovables (INER), analiza la naturaleza de funcionamiento del sector a nivel nacional, y se hace un primer acercamiento a los recorridos promedio del parque automotor ecuatoriano utilizando los despachos energéticos y la eficiencia por modo transporte (Díaz et al., 2014), sin embargo, este análisis no contabiliza las pérdidas y usos distintos del combustible, tales como contrabando, derrames, uso en talleres automotrices para lavado de partes, etc.

Olsson et al. (2015) y Kulisic et al. (2021) destacan la importancia de la implementación de políticas públicas relacionadas a la energía renovable en el transporte, puesto que las limitaciones y el bajo rendimiento de las tecnologías de vehículos disponibles que utilizan combustibles fósiles agravan cada vez más los problemas ambientales y de salud humana. Señalan que esto es por causa de que el $95 \%$ de la energía del transporte mundial proviene de combustibles derivados del petróleo, y este sector tiene la menor penetración de energía renovable. Además, mencionan que el transporte por carretera consume alrededor del $45 \%$ de la demanda mundial de petróleo, previendo que su participación seguirá siendo la misma para 2023 y que los vehículos con motor de combustión interna continuarán dominantes dentro de la flota vehicular mundial. Según SLoCat (2020), en Ecuador, el crecimiento de las emisiones de CO2 del transporte está superando el crecimiento económico. Si bien la tasa de motorización del país es muy alta, al mismo tiempo, en ciudades como Quito, más de la mitad de todos los viajes se realizan en transporte colectivo, lo que favorece la disminución de kilómetros recorridos por vehículos privados y por ende la contaminación. Además, reportan que la nación trabaja en la implementación de estrategias integrales de transporte, donde varias ciudades tienen planes ambiciosos para aumentar el transporte público y la infraestructura para caminar y andar en bicicleta. A nivel nacional, se está desarrollando un plan de movilidad urbana, pero el país actualmente carece de marcos suficientes para aumentar la eficiencia del transporte, como estándares de consumo de combustible y medidas para promover la electrificación. Bajo esta primicia, resulta imperioso desarrollar investigaciones respecto al indicador $\mathrm{KVR}$, que se deriven en aplicaciones para, por ejemplo, estimar el consumo energético del transporte, intensidad energética, volumen de tráfico, emisiones de $\mathrm{CO} 2$, así como analizar temas relacionados al funcionamiento global de los sistemas de transporte de una región.

Precisamente, el objetivo de esta investigación radica en desarrollar una nueva metodología de cálculo del indicador KVR para estimar el balance energético del sistema de transporte por carretera, que a diferencia de otros estudios considere los consumos no justificados. Sumado a ello, el estudio incluye la población total de vehículos registrada y clasificada por uso en la provincia de Loja, Ecuador, y tipo de combustible consumido. Que se haya considerado el parque automotor total de una ciudad, ayudaría a reflejar una densidad de tráfico que represente la realidad nacional. La hipótesis planteada en el presente estudio indica que con un adecuado método de cálculo se obtendrá una estimación conveniente del balance energético de transporte, donde la diferencia entre la estimación de consumos energéticos, mediante el uso de un KVR regional, no supere el $5 \%$ del despacho energético entregado por la Agencia de Regulación y Control Hidrocarburífera (ARCH). 


\section{METODOLOGÍA}

La metodología de cálculo, a partir del análisis de ventas de combustibles y de la clasificación del parque automotor para determinar los recorridos promedio e indicador KVR del presente estudio, hace referencia a los siguientes supuestos: a) El combustible despachado en las diferentes estaciones de servicio de la provincia de Loja son usados por el parque automotor como energía útil, en ese sentido los despachos pueden ser considerados como el consumo de energía del parque automotor provincial, alineado al criterio de OLADE de "Lo comprado es consumido". De esta manera, todo el combustible que no se usa para el trabajo útil de transporte se lo considera como consumos no justificados (CNJ) de energía o como energía no útil; b) Los recorridos promedio e indicadores KVR estimados para regiones cercanas al Ecuador son una referencia para el inicio de iteraciones de cálculo; c) El consumo de energía de los grupos de vehículos eléctricos y GLP, en la Provincia de Loja, son despreciables para el cálculo y en la aplicación del flujo de energía, debido al reducido número de unidades ( $0,24 \%$ del total de vehículos); y d) Se plantea la existencia de una analogía entre el análisis de flujo en conductos de Cross Hardy con el flujo de energía para el sistema de transporte (balance energético).

\section{Balance de energía sector transporte}

Para lograr hacer un cálculo de estimación de indicador KVR, después de haber analizado la información de población, parque automotor y despachos, se plantea un balance de energía útil para el combustible despachado para usos automotrices representado por la ecuación 1.

$$
E_{\text {entra }}-E_{\text {sale }}=\left(Q_{\text {entra }}-Q_{\text {sale }}\right)+\left(W_{\text {entra }}-W_{\text {sale }}\right)+\left(E_{\text {masa }, \text { entra }}-E_{\text {masa,sale }}\right)=\Delta E
$$

Donde $\mathrm{E}$ es la energía del sistema, $\mathrm{Q}$ es la transferencia de calor del sistema, W es el trabajo realizado dentro del sistema. Para el caso de estudio, se obtendría la ecuación 2.

$$
E_{\text {despachada }}=\left[\left(W_{\text {entra }}-W_{\text {sale }}\right)+C N J\right]
$$

En esta ecuación, $E_{\text {despachada }}$ es el combustible despachado en las estaciones de servicio, $W_{\text {entra }}-W_{\text {sale }}$ es el trabajo realizado por los vehículos para realizar sus desplazamientos de transporte utilizando la energía suministrada y $C N J$ es el consumo no justificado, es decir, no usado para transporte o pérdidas. Por otro lado, como en esta investigación se plantea la analogía existente entre el modelo de flujos en conductos de Hardy Cross y el flujo de energía en un sistema cerrado que corresponde al sector transporte terrestre, es viable la resolución del sistema cuando falten ecuaciones para la matriz de cálculo a través de iteraciones. El número de iteraciones necesarias para llegar al resultado esperado se da una vez que se obtenga un error menor al $5 \%$ como se planteó en la hipótesis alternativa. Se cree conveniente utilizar esta analogía, puesto que la energía puede idealizarse como un flujo tal como en un diagrama de Sankey, ya que es conocido el volumen energético entrante al sistema de transporte. Además, teniendo en cuenta que la energía no se pierde en el sistema, también es conocida la cantidad de energía de salida (trabajo más pérdidas).

El enfoque racional de Cross, es una técnica iterativa para determinar el flujo en sistema de tres o más tuberías, donde las entradas y las salidas de caudal son conocidas, pero el flujo dentro de la red es desconocido, por lo que aplica un balance hidráulico, es decir, se sabe el caudal de fluido que entra y es igual al fluido que sale del sistema. Se utiliza una estimación inicial que satisface la continuidad del flujo en cada unión y luego equilibra los flujos hasta que se consigue también la continuidad del potencial sobre cada bucle en el sistema. Esto debido a que el sistema de ecuaciones como tal no tiene solución, pues existen mayor número de incógnitas que ecuaciones; esto se lo solventa utilizando las aproximaciones sucesivas. Asimismo, para el flujo de energía usado en las actividades de transporte se conoce la entrada de energía, la salida en forma de trabajo de la maquinaria sumado a las pérdidas, pero la cantidad de energía usada en procesos internos de consumo y de conversión de energía en trabajo son desconocidos, por lo que pueden ser resueltas con iteraciones a partir de un balance de energía, como ya se indicó. De esta manera, el total de combustible despachado en las estaciones de servicio sería la energía de entrada. Para la construcción de la matriz de cálculo se recurre a las siguientes ecuaciones 3, 4 y 5 (Góngora, 2012).

$$
\begin{aligned}
& \sum_{0}^{n} E_{\text {total }}=\sum \frac{k v r_{1}}{E f_{1}}+\frac{k v r_{2}}{E f_{2}}+\frac{k v r_{3}}{E f_{3}}+\frac{k v r_{n}}{E f_{n}}+E_{C N J} \\
& k v r_{\text {vehiculos }}=\frac{E * E f}{N_{\text {vehiculos }}}
\end{aligned}
$$


$\sum_{0}^{n} E_{\text {total }}=\left(E D_{\text {autos }}+E D_{S U V^{\prime} s}+E G_{\text {autos } \ldots+E_{C N J}}\right)$

Donde $E_{\text {total }}$ es la energía del sistema analizado, Ef es la eficiencia promedio de un grupo vehicular y $N_{\text {vehículos }}$ es el número de vehículos de cada tipo. En la ecuación 5, ED es la energía en forma de diésel, EG es la energía en forma de gasolina, los subíndices $E G_{a u t o s}+E D_{S U V ' s}$ se refieren a la Energía calculada para cada grupo vehicular. En este cálculo, el flujo por las tuberías indicado por Cross Hardy se convertiría en el flujo imaginario de energía en un diagrama de Sankey.

\section{Desagregación del parque automotor}

En Ecuador existen varias instituciones nacionales que han clasificado el parque automotor, entre ellas el INEC, tal como se observa en la Fig. 2. El parque automotor de la provincia de Loja para 2014 está desagregado como indica la Fig. 3.

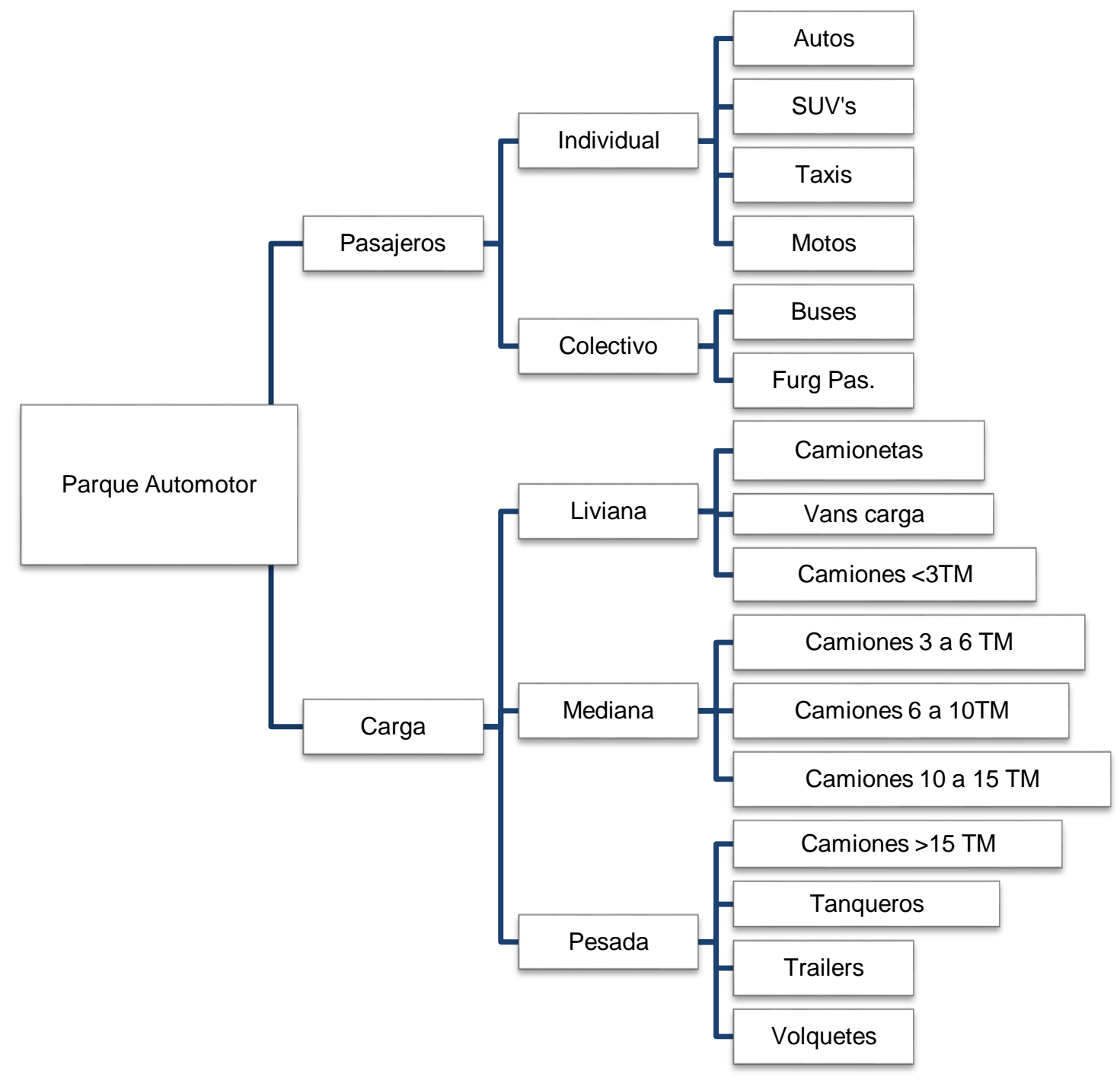

Fig. 2: Clasificación del parque automotor nacional. (Adaptada de INEC, 2015)

Se observa los porcentajes de desagregación según clasificación del número de vehículos motorizados matriculados, por uso y tipo de combustible. Existe un total de 45.464 vehículos, donde 39.238 utilizan gasolina, 6.118 diésel, 93 son híbridos, 12 usan GLP y 3 son eléctricos. A más de ello, se destaca la mayor presencia de autos con 13.808 unidades, seguido de 12.887 camionetas. Los vehículos con tecnología híbrida, GLP y eléctricos, como se observa, no son representativos para el cálculo de la investigación. Asimismo, la antigüedad del parque automotor juega un papel muy importante a la hora de calcular el consumo de energía de una flota. Así, tomando como año límite el 2014, un gran porcentaje de vehículos automotores en la provincia de Loja es relativamente nuevo, esto es el $40 \%$ con una edad menor a 5 años; el parque automotor con una edad mayor a 30 años corresponde solo al $10 \%$. 
La desagregación del parque automotor no tiene similitud al reparto modal de viajes realizados en la ciudad de Loja, que indica un bajo número de unidades para transporte público, esto a pesar de ser el medio de transporte más usado, dando a entender inequidad no sólo en inversión en infraestructura para transporte, sino también en el uso y ocupación del espacio público y los recursos energéticos. El parque automotor de transporte particular (automóviles) está conformado principalmente por vehículos a gasolina; el que realiza actividades de transporte público y transporte de carga, por vehículos a diésel. La clasificación "Otra clase" integra la maquinaria agrícola.

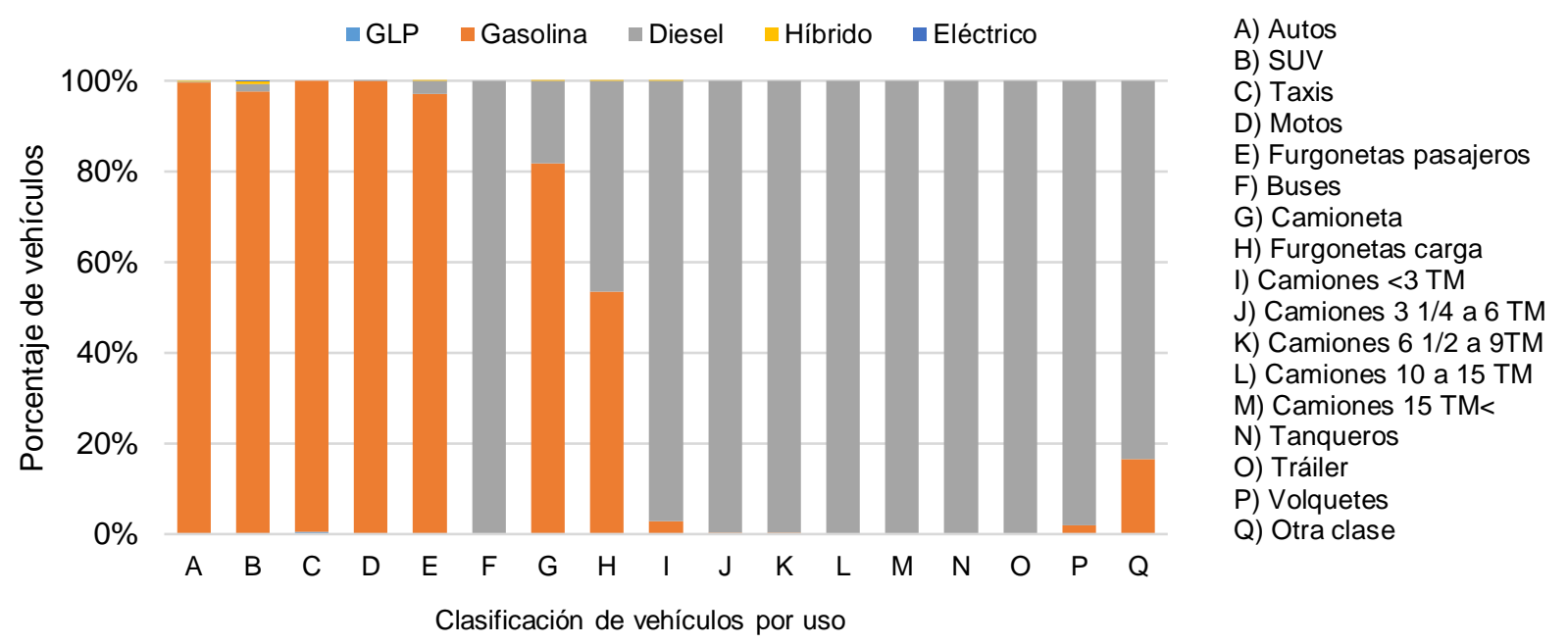

Fig. 3: Desagregación del parque automotor y tipo de combustible de la provincia de Loja. (Datos tomados de INEC, 2015)

\section{Eficiencia del parque automotor}

Según el informe de clasificación por consumo y eficiencia energética por modo de transporte generada, desarrollado por INER, indica que la eficiencia del parque automotor ecuatoriano está determinada como indica la Tabla 1 (Díaz et al., 2014). El procedimiento para estimar la eficiencia del parque automotor se basa en ciclos de conducción, considerando recorridos combinados (urbano-autopista) en la ciudad de Quito, realizados por el CCICEV-EPN (Centro de Transferencia Tecnológica para la Capacitación e Investigación en Control de Emisiones Vehiculares de la Escuela Politécnica Nacional).

Tabla 1: Eficiencia parque automotor nacional.

\begin{tabular}{|l|c|c|c|}
\hline & Gasolina & Diesel & Híbrido \\
\hline & {$[\mathrm{km} /$ gal $]$} & {$[\mathrm{km} / \mathrm{gal}]$} & {$[\mathrm{km} /$ gal $]$} \\
\hline Autos & 44,10 & 45,00 & 63,00 \\
\hline Suv & 31,74 & 43,00 & 35,00 \\
\hline Taxis & 44,10 & 44,00 & \\
\hline Motos & 30,00 & 35,00 & \\
\hline Furg. $P$ & 35,00 & 42,00 & \\
\hline Buses & 20,00 & 30,00 & \\
\hline Camionetas & 34,16 & 42,00 & 35,00 \\
\hline Furg. C & 25,00 & 30,00 & \\
\hline$<3$ TM & 20,00 & 25,00 & \\
\hline 3 1/4 a 6 TM & 18,00 & 22,00 & \\
\hline 6 1/2 a 9TM & 15,00 & 20,00 & \\
\hline 10 a 15 TM & 13,00 & 17,00 & \\
\hline 15 TM< & 6,00 & 12,00 & \\
\hline Tanqueros & 4,00 & 9,00 & \\
\hline Trailers & 6,00 & 10,00 & \\
\hline Volquetes & 4,00 & 9,00 & \\
\hline
\end{tabular}




\section{Análisis de ventas de combustible}

Se ha realizado análisis de ventas de combustible para la provincia de Loja en un periodo de ocho años (20072015), y se ha encontrado un importante incremento de uso de energía para transporte por carretera. En el 2014 se despacharon 1'223.198 BEP y para 2015 un total 1'178.738 BEP (ARCH, 2016). Para el análisis de ventas y consumo de combustible se usa al criterio de OLADE sobre las ventas de combustible, el cual indica que "lo comprado es consumido". La empresa pública Petroecuador es quien cuantifica y entrega los derivados de petróleo a las más de mil comercializadoras, desde sus refinerías, terminales y depósitos, atendiendo la demanda de la nación. Los despachos de combustible en las estaciones de servicio son mayoritariamente utilizados en usos automotrices, sin embargo, la presente investigación adiciona el criterio de cálculo de consumos no justificados o pérdidas en el sistema energético de transporte. En la Tabla 2 se observa los despachos mensuales por tipo de combustible en BEP en el 2014. Así, el $51 \%$ de despachos se refiere a diésel con $616 \mathrm{kBEP}$, gasolina Extra un 44\% con $549 \mathrm{kBEP}$; y, sólo un $5 \%$ de gasolina Súper con $57 \mathrm{kBEP}(\mathrm{ARCH}, 2016)$.

Tabla 2: Despachos mensuales por tipo de combustible en BEP

\begin{tabular}{|l|c|c|c|c|}
\hline & Diesel Premium & Gas. Extra & Súper S.P. & Total \\
\hline Enero & 50.704 & 45.288 & 5.075 & 101.067 \\
\hline Febrero & 47.871 & 42.267 & 4.208 & 94.346 \\
\hline Marzo & 48.509 & 44.606 & 4.318 & 97.432 \\
\hline Abril & 48.568 & 43.421 & 4.569 & 96.558 \\
\hline Mayo & 51.672 & 46.379 & 4.572 & 102.623 \\
\hline Junio & 46.136 & 42.603 & 3.935 & 92.674 \\
\hline Julio & 51.506 & 46.683 & 4.610 & 102.799 \\
\hline Agosto & 55.543 & 48.173 & 5.768 & 109.484 \\
\hline Septiembre & 53.867 & 47.953 & 5.328 & 107.147 \\
\hline Octubre & 55.413 & 48.351 & 5.395 & 109.159 \\
\hline Noviembre & 51.176 & 45.131 & 4.399 & 100.705 \\
\hline Diciembre & 55.094 & 48.844 & 5.265 & 109.204 \\
\hline Total & 616.059 & 549.697 & 57.442 & 1223.198 \\
\hline
\end{tabular}

En la Fig. 4 se verifica la evolución de despachos de Diésel y Gasolina para el mismo período de análisis. Se evidencia una proporción de despachos en partes iguales de ambos combustibles. Realizar esta clasificación y tener únicamente dos tipos de combustibles facilita el cálculo y permite analizar de mejor manera cómo se comportan los consumos energéticos en el sector transporte según el tipo de combustible.

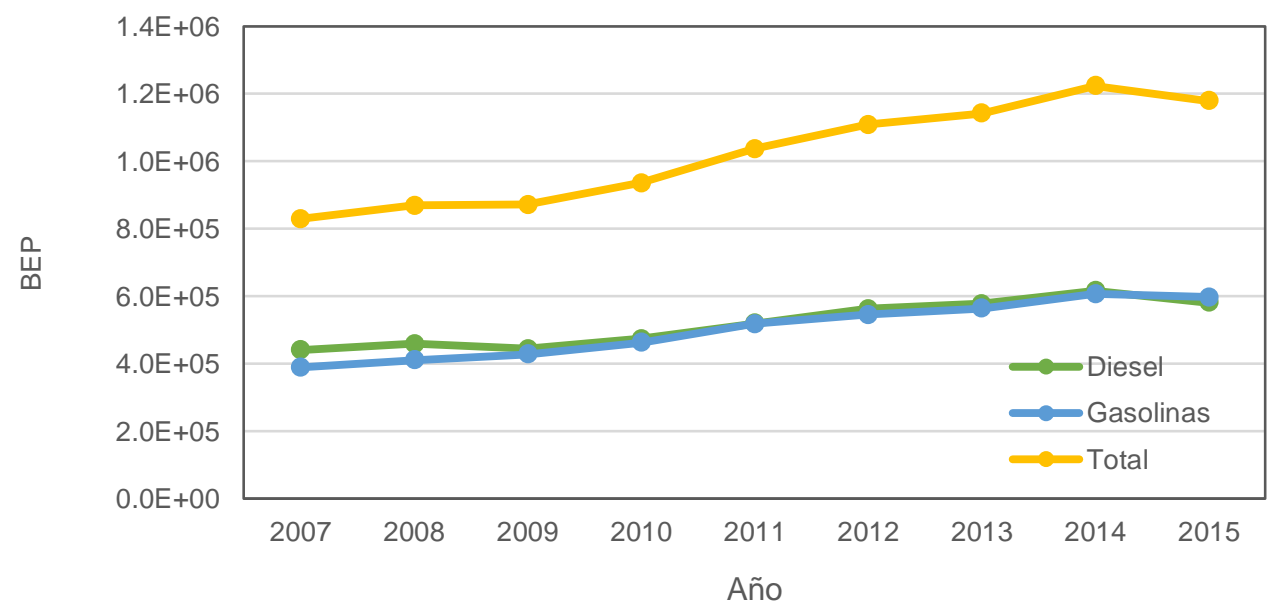

Fig. 4: Comparación de despachos de combustible Diésel y Gasolina (Datos tomados de ARCH, 2016)

Sólo en el caso del Diésel en 2009 se observa una leve caída respecto al 2008, en todos los otros casos, en años subsiguientes se tiene un incremento en los despachos. En el diagrama de áreas de la Fig. 5 se observa los despachos por tipo de combustible para transporte en el periodo estudiado. Las tasas de crecimiento en los despachos de combustible se resumen en la Tabla $3(\mathrm{ARCH}, 2016)$; se aprecia que la gasolina extra presenta la mayor tasa de crecimiento, tanto en el período 2007-2014, como anualmente. 


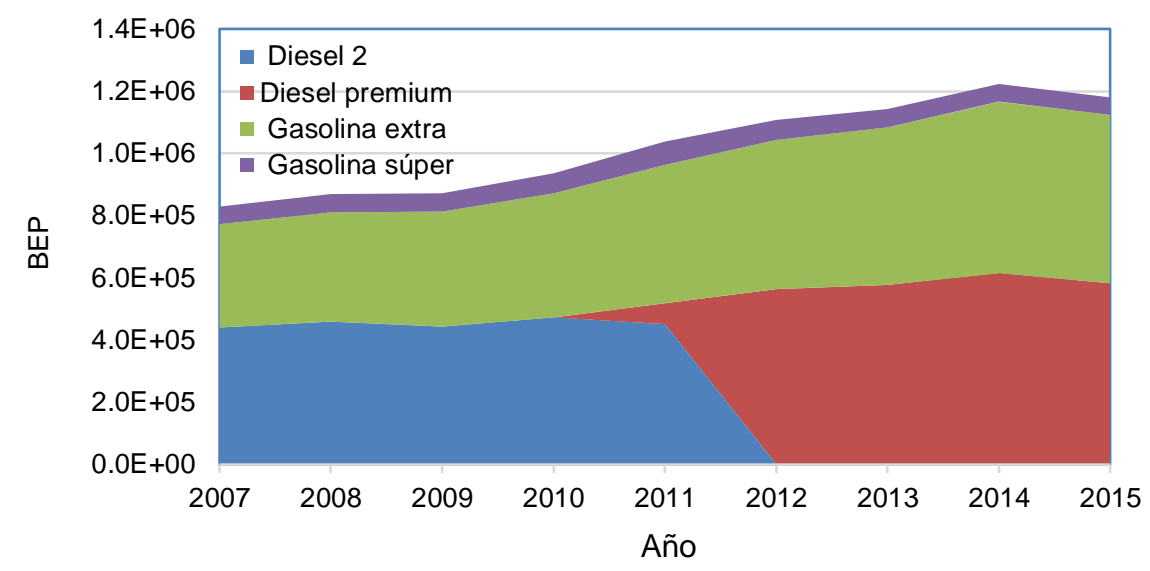

Fig. 5: Despachos por tipo de combustibles 2007-2015 (Adaptada de ARCH, 2016)

Tabla 3: Tasas de crecimiento de despacho de combustible

\begin{tabular}{|l|c|c|}
\hline & Período 2007-2014 & Promedio anual \\
\hline Diesel & $39,91 \%$ & $4,29 \%$ \\
\hline Gas. Extra & $65,40 \%$ & $6,49 \%$ \\
\hline Super s.p. & $2,31 \%$ & $0,38 \%$ \\
\hline Gasolinas & $56,01 \%$ & $5,72 \%$ \\
\hline Todos combustibles & $47,46 \%$ & $4,97 \%$ \\
\hline
\end{tabular}

\section{RESULTADOS}

La Tabla 4 muestra, por un lado, los resultados finales de consumo de combustible que coinciden con los datos de despacho de $(\mathrm{ARCH}, 2016)$. La diferencia entre el consumo calculado y la energía despachada tiene un error del $2 \%$. Los recorridos promedio ingresados en la matriz de cálculo en cada ecuación lineal son los más cercanos a la realidad de consumo de energía para cada grupo. Por otro lado, la Tabla 4, también presenta los recorridos promedio calculados para el año 2014. Se recalca que la solución a este sistema de ecuaciones, para verificar el flujo de energía, hasta este punto no contempla los gastos energéticos generados por pérdidas, es decir, estos recorridos suponen que todos los vehículos están trabajando los 365 días del año sin parar. Sin embargo, la realidad es otra, pues los vehículos tienen paros debido a circunstancias previstas o imprevistas: mantenimiento, días de ocio, vacación, accidentes, etc.

Tabla 4: Resultados del cálculo final del consumo de combustible y KVR en la provincial de Loja

\begin{tabular}{|c|c|c|c|c|}
\hline & \multicolumn{3}{|c|}{ Cálculo del consumo de combustible [gal] } & \multirow{2}{*}{$\begin{array}{l}\text { Cálculo de } \\
\text { KVR [km] }\end{array}$} \\
\hline & Gasolina & Diesel & Hibrido & \\
\hline Autos & $5.828 .564,0$ & $11.640,16$ & 799,11 & 18.707 \\
\hline Suv & $4.460 .542,00$ & $52.206,70$ & $30.466,34$ & 18.707 \\
\hline Taxis & $1.077 .187,01$ & & & 78.389 \\
\hline Motos & $3.085 .106,36$ & 438,10 & & 15.334 \\
\hline Furg. P & $389.814,27$ & $9.936,44$ & & 32.102 \\
\hline Buses & $15.050,92$ & $682.308,20$ & & 75.255 \\
\hline Camionetas & $11.970 .832,50$ & $2.176 .050,53$ & $3.329,27$ & 38.842 \\
\hline Furg. C & $604.958,17$ & $437.296,15$ & & 57.288 \\
\hline$<3$ TM & $90.995,41$ & $2.500 .273,95$ & & 69.996 \\
\hline $31 / 4$ a 6 TM & $49.726,59$ & $4.897 .503,60$ & & 111.885 \\
\hline $61 / 2$ a 9TM & $11.864,78$ & $806.804,85$ & & 59.324 \\
\hline 10 a 15 TM & & $417.382,65$ & & 55.004 \\
\hline $15 \mathrm{TM}<$ & & $365.407,34$ & & 69.601 \\
\hline Tanqueros & & $265.966,21$ & & 53.193 \\
\hline Trailers & & $3.009 .081,13$ & & 189.250 \\
\hline Volquetes & $423.742,57$ & $9.876 .863,85$ & & 188.330 \\
\hline Otros & $80.938,56$ & $181.576,44$ & & 15.417 \\
\hline Subtotal & $28.089 .323,22$ & $25.690 .736,31$ & $43.594,73$ & \\
\hline Total calc. & 28.132.917,95 & $25.690 .736,31$ & & \\
\hline Total desp. & $28.541 .610,00$ & $25.837 .515,00$ & & \\
\hline$\Delta$ Variación & $408.692,05$ & $146.778,69$ & & \\
\hline Error & $1,43 \%$ & $0,57 \%$ & & \\
\hline
\end{tabular}




\section{Cálculo de usos no justificados en el flujo de energía}

El cómputo anterior genera una solución al sistema de ecuaciones lineales aplicando el método Hardy Cross. Sin embargo, hasta el momento, para este resultado se entiende que los 365 días del año los vehículos están circulando y que la energía del sistema se usa para generar el trabajo útil del parque automotor global sin pérdidas. Pero la realidad es diferente, pues el parque automotor no funciona todo el año y cierta parte de la energía se pierde en derrames de combustibles, usos en talleres de mecánica, contrabando en la zona fronteriza, etc. Esta diferencia, entre la energía usada y las pérdidas, está directamente relacionada con la intensidad de uso de los vehículos, es decir, cuántos días al año éstos están parados. Con esta primicia, es preciso en este punto hacer una nueva hipótesis al uso de la energía en transporte, referente al tiempo anual de operación de los vehículos. El estudio del cálculo de emisiones para los proyectos de transporte realizado por Ferreira et al. (2012), sirve de referencia para estimar las pérdidas debido a que no se tiene información local. Además, plantea que el uso promedio de la operación de los vehículos en un año es de 320 días para los de uso familiar y de 350 días para vehículos de trabajo, teniendo en cuenta que todos los vehículos en algún momento tienen periodos de paro, ya sea por mantenimiento, vacaciones o simplemente los usuarios utilizan otros medios de movilización. En términos de tiempo se calcula un factor de uso de toda la flota de vehículos en un 0,88 para los familiares y 0,96 para los comerciales, tal como se enlista en la Tabla 5.

Tabla 5: Factor de uso de los grupos vehiculares

\begin{tabular}{|l|c|}
\hline Tipo de Vehículo & Factor de uso \\
\hline Vehículos de trabajo & 0,88 \\
\hline Vehículos particulares & 0,88 \\
\hline Vehículos comerciales & 0,96 \\
\hline Promedio & 0,91 \\
\hline
\end{tabular}

Se aplica el factor de uso así: 0,88 para autos, SUV's y motos; para taxis, furgonetas, buses, camiones livianos y medianos 0,96; y finalmente se aplica el promedio a camionetas, y a los vehículos de carga pesada 0,92. Con esta modificación el gasto energético queda calculado como se muestra en la Tabla 6, donde el total es de 1'116.633,10 BEP. Además, los consumos no justificados o pérdidas están representadas por la diferencia entre la magnitud de energía total calculada $E_{t c}$ menos el producto de la energía total calculada por el factor de uso FU. Por tanto, la estimación de las pérdidas responde a la ecuación 6 . En la misma Tabla 6 se muestra el resultado de la cantidad de energía despachada cuyo uso no está justificado, esto es 106.565,03 BEP.

$$
L=E_{t c}(1-F U)
$$

Tabla 6: Cálculo de consumo de energía y KVR anual con factor de uso; uso no justificado de energía por grupo vehicular.

\begin{tabular}{|c|c|c|c|c|c|c|c|c|}
\hline & \multirow{2}{*}{$\begin{array}{l}K V R \\
{[\mathrm{~km}]}\end{array}$} & \multicolumn{3}{|c|}{ Consumo de energía [BEP] } & \multicolumn{4}{|c|}{ Uso no justificado [BEP] } \\
\hline & & Gasolina & Diesel & Hibrido & Gasolina & Diesel & Hibrido & Total \\
\hline Autos & 16.463 & 110205,94 & 246,70 & 185,28 & 15028,08 & 33,64 & 25,27 & 15086,99 \\
\hline Suv & 16.463 & 84339,50 & 1106,45 & 576,05 & 11500,84 & 150,88 & 78,55 & 11730,27 \\
\hline Taxis & 75.254 & 22218,93 & 0,00 & 0,00 & 925,79 & 0,00 & 0,00 & 925,79 \\
\hline Motos & 13.494 & 58332,90 & 9,28 & 0,00 & 7954,49 & 1,27 & 0,00 & 7955,75 \\
\hline Furg. P & 30.818 & 8040,62 & 229,73 & 0,00 & 335,03 & 9,57 & 0,00 & 344,60 \\
\hline Buses & 72.244 & 310,45 & 15775,18 & 0,00 & 12,94 & 657,30 & 0,00 & 670,23 \\
\hline Camionetas & 35.734 & 236631,70 & 48214,69 & 65,81 & 20576,67 & 4192,58 & 5,72 & 24774,97 \\
\hline Furg. C & 54.996 & 12478,35 & 10110,43 & 0,00 & 519,93 & 421,27 & 0,00 & 941,20 \\
\hline$<3 \mathrm{TM}$ & 64.397 & 1798,74 & 55398,50 & 0,00 & 156,41 & 4817,26 & 0,00 & 4973,67 \\
\hline 3 1/4 a 6 TM & 102.934 & 982,96 & 108513,85 & 0,00 & 85,48 & 9435,99 & 0,00 & 9521,46 \\
\hline $61 / 2$ a 9TM & 54.578 & 234,54 & 17876,35 & 0,00 & 20,39 & 1554,47 & 0,00 & 1574,86 \\
\hline 10 a 15 TM & 50.604 & 0,00 & 9247,94 & 0,00 & 0,00 & 804,17 & 0,00 & 804,17 \\
\hline $15 \mathrm{TM}<$ & 64.033 & 0,00 & 8096,32 & 0,00 & 0,00 & 704,03 & 0,00 & 704,03 \\
\hline Tanqueros & 48.938 & 0,00 & 5893,01 & 0,00 & 0,00 & 512,44 & 0,00 & 512,44 \\
\hline Trailers & 174.110 & 0,00 & 66672,12 & 0,00 & 0,00 & 5797,58 & 0,00 & 5797,58 \\
\hline Volquetes & 173.264 & 8376,27 & 218841,39 & 0,00 & 728,37 & 19029,69 & 0,00 & 19758,06 \\
\hline Otros & 14.184 & 1599,94 & 4023,18 & 0,00 & 139,13 & 349,84 & 0,00 & 488,97 \\
\hline Total & --- & 545550,84 & 570255,11 & 827,15 & 57983,54 & 48471,95 & 109,54 & 106565,03 \\
\hline
\end{tabular}




\section{Validación de Resultados}

Para realizar una validación de los resultados se ha creído pertinente hacer una comparación de recorridos con los efectuados por el transporte de taxi de la ciudad de Loja, esto a través del levantamiento de una encuesta dirigida a conductores de varias compañías, entre las cuales constan: Cooperativa de Taxis La Pradera, Terminal Terrestre, Julio Ordoñez, entre otras. La información recopilada colecciona los datos de odómetro actual y se consulta a 40 propietarios de taxis los recorridos diarios realizados.

\section{Consumos energéticos de transporte en la provincia de Loja}

Los resultados de consumo de energía, y pérdidas, por uso de vehículos se muestran en las Fig. 6 y 7 . De un total de 607 mil BEP de gasolina la distribución de su consumo se muestra en la Fig. 6, donde predomina los vehículos de pasajeros individual con un $45,64 \%$, seguido muy de cerca por los vehículos de carga liviana con un $41,52 \%$.

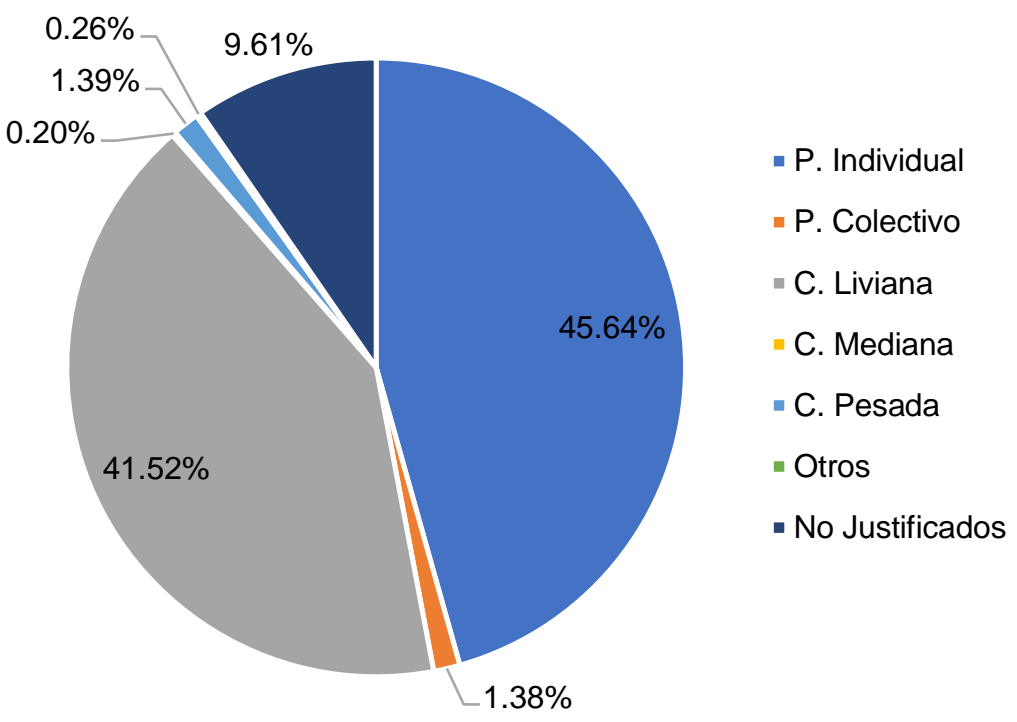

Fig. 6: Distribución de consumo de gasolina en la provincia de Loja

En la Fig. 7 se refleja la distribución del consumo de un total de 616 mil BEP de diésel. Predomina el consumo para vehículos de carga pesada con casi la mitad del total de diésel distribuido, esto es $48,41 \%$, seguido por los de carga mediana con un $21,92 \%$.

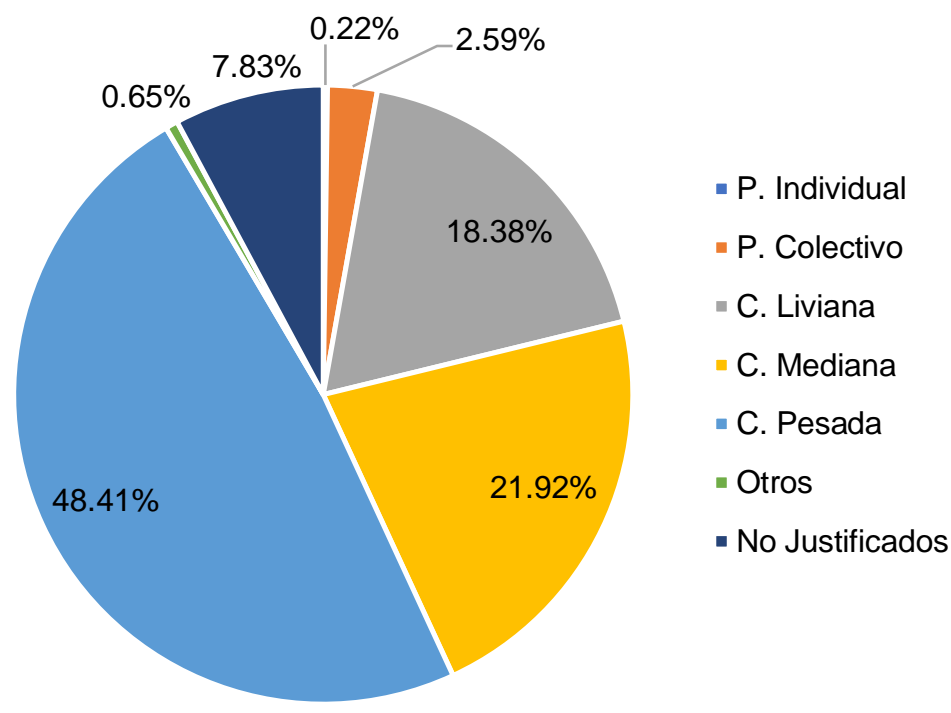

Fig. 7: Distribución de consumo de Diésel en la provincia de Loja 


\section{Consumo total de energía}

Finalmente, para el total de la energía consumida en la provincia de Loja (diésel y gasolina) de 1'223.198 BEP, el consumo queda repartido según indica la Fig. 8 . Se observa que el $22,66 \%$ corresponde a vehículos de pasajeros individual y solo un $2 \%$ para transporte masivo de pasajeros; esto claramente indica una movilidad poco sostenible. Además, existe un predominio de la energía consumida para vehículos de carga liviana, esto es $29,82 \%$, respecto a los vehículos de carga mediana y pesada, con $11,19 \%$ y $25,17 \%$, respectivamente. Finalmente, se calculó un $8,71 \%$ de la energía en usos no justificados.

\section{Diagrama de flujo de la energía}

La investigación sobre el diagrama de Sankey para flujos de energía y exergía realizada por Soundararajan et. al. (2014), indica que éstos se han utilizado como una herramienta eficaz para centrarse en el flujo de energía y su distribución en varios sistemas de energía, proporcionando una posible alineación de cómo se calculan y representan las pérdidas de energía, identificando áreas potenciales para mejoras en el rendimiento energético. A través de una revisión exhaustiva de los usos históricos de los diagramas de Sankey destacan su creciente importancia en la toma de decisiones y las políticas públicas. Finalmente, manifiestan que los flujos de energía constituyen las partes más básicas e importantes de un diagrama de Sankey y, en una determinada región, muestran las relaciones y los flujos desde los recursos energéticos (etapa de suministro primario) hasta el uso final (etapa de consumo). Bajo la luz de esto, es precisa su inclusión para mostrar los resultados del flujo energético del presente estudio, dada la naturaleza del mismo.

Así, en la Fig. 9 se presenta el diagrama de flujo (diagrama de Sankey) de energía final calculado. Éste permite observar cómo se comporta la energía en el sistema cerrado de transporte en la provincia de Loja. Se evidencia el flujo de energía útil en color magenta, que corresponde a 1'116.633,10 BEP, y las pérdidas de energía en color negro, esto es 106.565,03 BEP. Sumadas las dos salidas del sistema se tiene la energía total de entrada que es de 1'223.198 BEP, donde 618.721,10 BEP corresponde a combustible diésel y $604.471,1$ BEP a nafta; el ancho de las flechas representa la magnitud del caudal. Al ver el diagrama se aprecia diáfanamente cuál es la analogía entre un sistema de tuberías, como se indicó en la metodología, y la idealización de la energía como un fluido.

Por otro lado, aunque no forma parte de la presente investigación, es preciso mencionar que la energía útil todavía experimenta pérdidas inevitables que se producen por el funcionamiento peculiar de los motores de combustión interna, donde el rendimiento efectivo se ve condicionado por las pérdidas térmicas, el trabajo que dispone para el accionamiento de accesorios, pérdidas por bombeo que experimenta el motor en sus fases de renovación de carga, y el rozamiento al que están sujetos sus elementos mecánicos.

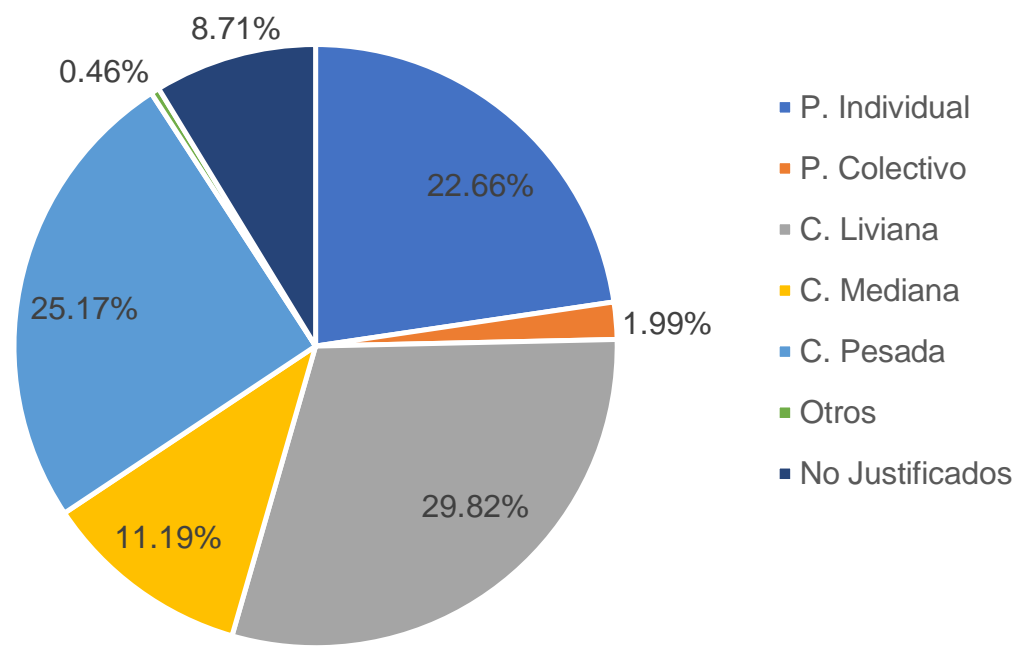

Fig. 8: Distribución de consumo total de energía para transporte en la provincia de Loja

\section{DISCUSIÓN}

En primer lugar, el análisis del crecimiento, tanto del parque automotor de la provincia como del consumo de energía para el sector transporte, indican la existencia de una relación directamente proporcional entre estas dos variables, y ninguna de estas se correlaciona con el crecimiento de la población. Es decir, la población 
crece a un ritmo mucho más lento que el parque automotor y los consumos de combustible. En segundo lugar, se evidencia la importancia de tener una metodología nacional para calcular el indicador KVR anualmente, y la generación de estudios sobre este indicador, tales como: (Góngora, 2012; Kumapley y Fricker, 1996), entre otros. Los datos presentados por el Balance Energético Nacional 2014 fueron generados a partir de un recorrido promedio de $20.000 \mathrm{~km}$ de automóviles (MICSE, 2014), esto se justifica por dos razones, la primera es que los datos de recorridos tomados en concesionarios automotrices en Ecuador realizan los cálculos de garantía de sus vehículos justamente con este kilometraje, y segundo, que a partir del cálculo de iteraciones se aproxima bastante a la realidad nacional de consumos y a la información de despachos presentada por la $\mathrm{ARCH}$, por lo que esta metodología empleada por el MICSE en líneas generales cumplió satisfactoriamente. Sin embargo, tuvo limitaciones tales como: no contabilizar las pérdidas en el sistema de distribución y consumo de energía del sector transporte; no considerar el factor de uso de los vehículos, pues se asume que éstos funcionan 365 días al año; y, no generan un indicador promedio KVR, sino únicamente un indicador de consumos.

Por otro lado, la metodología desarrollada en la presente investigación es más robusta debido a que considera las limitaciones mencionadas en estudios previos, y muestra una alternativa apropiada para el cálculo de consumos no justificados en el sistema energético del transporte. A pesar del avance en este sentido, y el hecho de incluir las pérdidas y el factor de uso automotriz, esta metodología presenta ciertos inconvenientes: 1) El factor de uso automotriz fue tomado del estudio de Ferreira et al. (2012), aunque es la información más cercana, puede ser mejorada con el levantamiento de datos propios en las regiones estudiadas; 2) No toma en cuenta el incremento de la densidad urbana; 3) No se tiene información precisa para validar los cálculos realizados, por lo que se torna necesario contar con estadísticos base en cada región analizada.

Las limitaciones mencionadas en los dos últimos ítems también lo experimentan la metodología empleada por el INER. Además, los vehículos con tecnología híbrida, GLP y eléctricos, han sido excluidos, puesto que no son representativos para el cálculo debido a su reducida población $(0,24 \%$ del total de vehículos en la zona de estudio). Por otro lado, el KVR en el presente estudio es utilizado para la estimación de los consumos energéticos de transporte, más no para determinar sus emisiones contaminantes en carretera, que bien podría ser un estudio futuro.

Es importante destacar que tener un primer acercamiento a las pérdidas de energía en el sistema de transporte aporta significativamente a los métodos revisados, tales como: (Gargett et al., 2011; Góngora, 2012; Kumapley y Fricker, 1996). Por otra parte, los cálculos de consumo de energía comparados con metodologías como: (Liu et al., 2013; Solís y Sheinbaum, 2013; Karathodorou et al., 2010), aunque presentan enfoques diferentes como el análisis de la densidad urbana, todas precisan tener ya calculado el indicador KVR, lo que reitera la necesidad de obtener estos datos anualmente como política pública.

A diferencia del presente estudio que considera los vehículos registrados, Jung et al. (2017) estiman el KVR utilizando el volumen de tráfico urbano centrándose en el transporte de mercancías, puesto que tienen la finalidad adicional de ponderar las emisiones contaminantes móviles en carretera. Sus hallazgos demuestran que el KVR de los vehículos registrados es 2,11 veces mayor que el de los volúmenes de tráfico aplicados, justificando que consideran para la estimación las carreteras principales y secundarias en el área objetivo, supeditada de las estaciones de monitoreo de tráfico, lo cual resulta una limitante. Además, su investigación no incluye el consumo energético del sistema de transporte por carretera.

Sierra (2016) propone una metodología para estimar el KVR y el consumo de combustible del transporte por carretera diferente a la del presente estudio. Aplica metodologías basadas en la medición de tráfico, específicamente, calcula el número exacto de kilómetros recorridos por un grupo de vehículos en un período de tiempo definido, verificando el valor registrado por el odómetro. No obstante, el tamaño de la muestra de vehículos es pequeña y no se considera representativa en relación con el total de la flota de la ciudad de Quito, lugar del estudio. Más aún, las localidades donde se efectuaron las pruebas de campo, no reflejan una densidad de tráfico que representa con precisión la realidad nacional. Sin embargo, la metodología que propone tiene factores en común respecto a la presente, tales como: la población de vehículos clasificada por uso en la sociedad, kilómetros recorridos y tipo de combustible consumido.

El funcionamiento de los sistemas de transporte tiene cierta complejidad para ser representado, esto se debe a que son fenómenos dinámicos y que no dependen de un solo operador. En el caso del flujo de energía para transporte, el tamaño de la flota vehicular, el consumo específico promedio y el KVR son características que limitan la cantidad de energía que puede fluir por cada subsistema. Desde el punto de vista de la solución del problema, la analogía del modelo Hardy Cross, junto con el balance energético de transporte, aportan con las herramientas adecuadas para generar los datos de KVR, a partir del cual se puede calcular los consumos energéticos para este sector. 


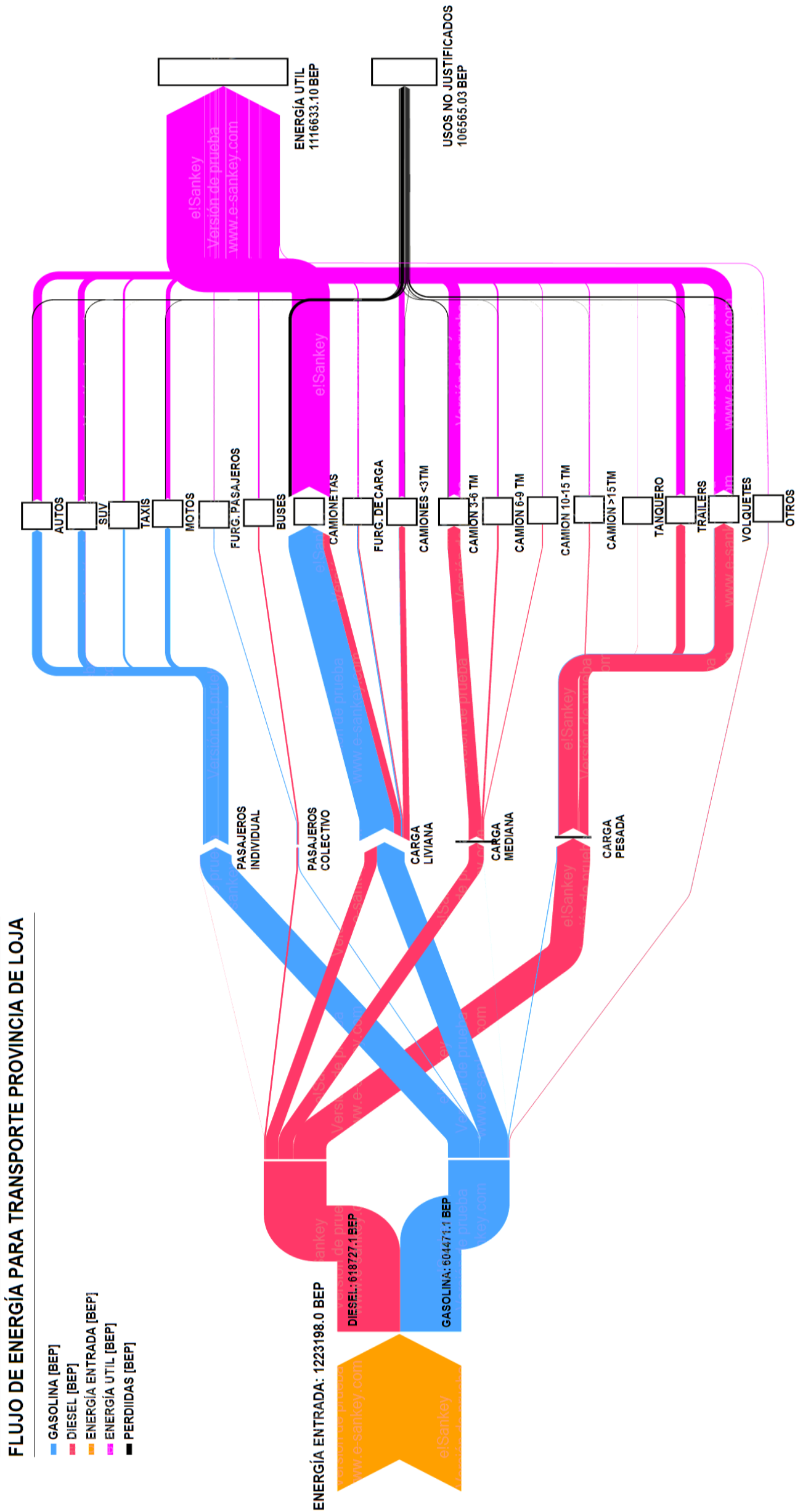

Fig. 9: Flujo de Energía para transporte en la Provincia de Loja 


\section{CONCLUSIONES}

A partir de los resultados obtenidos y a la discusión sobre distintos aspectos de la investigación, se pueden extraer las siguientes conclusiones: 1) El cálculo de los consumos de energía y uso final muestra que de un total de 1'123.198 BEP despachados para la provincia de Loja, un $24,65 \%$ es destinado para transporte de pasajeros y el $66,18 \%$ corresponde a actividades de transporte de carga; 2) La metodología es aplicable a la realidad nacional y puede ser replicable a otras provincias, puesto que las variaciones entre la energía consumida y la energía total despachada presentaron un error del 2\%; 3) Existe un consumo de energía del $8,71 \%$ que no está justificado y está relacionado con un parque automotor que no funciona todo el año, derrames de combustibles, usos en talleres de mecánica, contrabando en la zona fronteriza, entre otros; 4) Los KVR están condicionados por un factor de uso, donde los tráilers, con $174.110 \mathrm{~km}$, es el grupo vehicular que presenta mayor recorrido, por otro lado, los taxis con $75.254 \mathrm{~km}$ son los vehículos livianos que más recorren.

\section{REFERENCIAS}

ARCH., Despachos de combustibles 2014-2016, Agencia de Regulación y Control Hidrocarburífero, Quito (2016)

Bekka, A., Louvet, N., y Adoue, F., Impact of a ridesourcing service on car ownership and resulting effects on vehicle kilometers travelled in the Paris region, doi: 10.1016/j.cstp.2020.04.005, Case Studies on Transport Policy, 8(3), 10101018 (2020)

Díaz, J. P., Corral, A., y otros dos autores, Clasificación por consumo y eficiencia energética por modo de transporte generada, Linea Base para las Investigaciones en Eficiencia Energética en el Sector Transporte en Ecuador-INER (2014)

Engo, J., Decoupling analysis of CO2 emissions from transport sector in Cameroon, doi: 10.1016/j.scs.2019.101732, Sustainable Cities and Society, 51(1), 1-10 (2019)

Ferreira, L., Martinez, H., y otros dos autores, Gases de efecto invernadero en el subsector de transporte carretero en México (2012)

Figliozzi, M., Analysis of the efficiency of urban commercial vehicle tours: data collection, methodology, and policy implications, doi: 10.1016/j.trb.2007.04.006, Transportation Research Part B, 41(9), 1014-1032 (2007)

Gargett, D., Afzal, H., y Cosgrove, D., Road vehicle-kilometres travelled: estimation from state and territory fuel sales, Australian Government: Department of Infraestructure and Transport, BITRE (2011)

Góngora, J. P., Indicador kilómetros-vehículo recorridos (KVR), métodos de cálculo en diferentes países, Instituto de Políticas para el Transporte y el Desarrollo (2012)

IEA., Statistics: world energy balances 2018 overview, Paris (2018)

INEC., Anuario de estadísticas de transporte 2014, Quito (2015)

Jung, S., Kim, J., y otros tres autores, An estimation of vehicle kilometer traveled and on-road emissions using the traffic volume and travel speed on road links in Incheon city, doi: 10.1016/j.jes.2015.12.040, Journal of Environmental Sciences, 54(1), 90-100 (2017)

Karathodorou, N., Graham, D. J., y Noland, R. B., Estimating the effect of urban density on fuel demand, doi: 10.1016/j.eneco.2009.05.005, Energy Economics, 32(1), 86-92 (2010)

Kulisic, B., Dimitriou, I., y Mola-Yudego, B., From preferences to concerted policy on mandated share for renewable energy in transport, doi: 10.1016/j.enpol.2021.112355, Energy Policy, 155, 112355 (2021)

Kumapley, R. K., y Fricker, J. D., Review of methods for estimating vehicle miles traveled, doi: 10.1177/0361198196155100108, Transportation Research Record, 1551(1), 59-66 (1996)

Liu, Y., Wang, Y., y Huo, H., Temporal and spatial variations in on-road energy use and CO2 emissions in China, 19782008, doi: 10.1016/j.enpol.2013.05.121, Energy Policy, 61(1), 544-550 (2013)

Ministerior de Energía y Recursos Naturales no Renovables (MERNNR), Balance Energético Nacional (2019)

MICSE., Balance energético nacional (2014)

McIntosh, J., Trubka, R., y otros dos autores, The role of urban form and transit in city car dependence: analysis of 26 global cities from 1960 to 2000, doi: 10.1016/j.trd.2014.08.013, Transportation Research Part D, 33(1), 95-110 (2014)

Olsson, L., Hjalmarsson, L., y otros dos autores, Bridging the implementation gap: combining backcasting and policy analysis to study renewable energy in urban road transport, doi: 10.1016/j.tranpol.2014.10.014, Transport Policy, 37(1), 72-82 (2015)

Pérez, P., y Monzón de Cáceres, A., Consumo de energía por el transporte en España y tendencias de emisión, Observatorio Medioambiental, 11(1), 127-147 (2008)

Rehermann, F., y Romero, M. P., Economic growth and transport energy consumption in the latin american and caribbean countries, doi: 10.1016/j.enpol.2018.08.006, Energy Policy, 122(1), 518-527 (2018) 
Rocha-Hoyos, J., Tipanluisa, L., y otros dos autores, Estudio de un motor a gasolina en condiciones de altura con mezclas de aditivo orgánico en el combustible, doi: 10.4067/S0718-07642018000500325, Información Tecnológica, 29(5), 325-334 (2018)

Seethaler, R., y Rose, G., Using odometer readings to assess VKT changes associated with a voluntary travel behaviour change program, doi.org/10.1016/j.tranpol.2009.10.006, Transport Policy, 16(6), 325-334 (2009)

Shimoda, Y., Yamaguchi, Y., y otros seis autores, Energy demand science for a decarbonized society in the context of the residential sector, doi: 10.1016/j.rser.2020.110051, Renewable and Sustainable Energy Reviews, 132(1), 1-13 (2020)

SLoCaT., Transport and Climate Change Global Status Report (2018)

Sierra, J. C., Estimating road transport fuel consumption in Ecuador, doi: 10.1016/j.enpol.2016.02.008, Energy Policy, 92(1), 359-368 (2016)

Solís, J. C., y Sheinbaum, C., Energy consumption and greenhouse gas emission trends in mexican road transport, doi: 10.1016/j.esd.2012.12.001, Energy for Sustainable Development, 17(3), 280-287 (2013)

Song, M., Wu, N., y Wu, K., Energy consumption and energy efficiency of the transportation sector in Shanghai, doi: 10.3390/su6020702, Sustainability, 6(2), 702-717 (2014)

Weerasekera, T. D., y Amarasingha, N., Estimation of vehicle kilometers travelled in southern province, Sri Lanka, doi: 10.1109/nctm.2017.7872825, 6th National Conference on Technology and Management (NCTM), 40-45 (2017) 
